\title{
Cell Migration Induction
}

National Cancer Institute

\section{Source}

National Cancer Institute. Cell Migration Induction. NCI Thesaurus. Code C40743.

Cell Migration Induction involves initiation of active directed translocation of a whole cell, or cell body, from one site to another in response to a gradient. (NCI) 